\title{
Model Theory for Sheaves of Modules
}

DOI:

10.1007/978-3-662-58771-3_9

\section{Document Version}

Accepted author manuscript

Link to publication record in Manchester Research Explorer

\section{Citation for published version (APA):}

Prest, M. (2019). Model Theory for Sheaves of Modules. In Lecture Notes in Computer Science (Vol. 11600, pp. 89-102). [Chapter 9] (Logic and Its Applications; Vol. 11600). Springer Nature. https://doi.org/10.1007/978-3-66258771-3_9

\section{Published in:}

Lecture Notes in Computer Science

\section{Citing this paper}

Please note that where the full-text provided on Manchester Research Explorer is the Author Accepted Manuscript or Proof version this may differ from the final Published version. If citing, it is advised that you check and use the publisher's definitive version.

\section{General rights}

Copyright and moral rights for the publications made accessible in the Research Explorer are retained by the authors and/or other copyright owners and it is a condition of accessing publications that users recognise and abide by the legal requirements associated with these rights.

\section{Takedown policy}

If you believe that this document breaches copyright please refer to the University of Manchester's Takedown Procedures [http://man.ac.uk/04Y6Bo] or contact uml.scholarlycommunications@manchester.ac.uk providing relevant details, so we can investigate your claim.

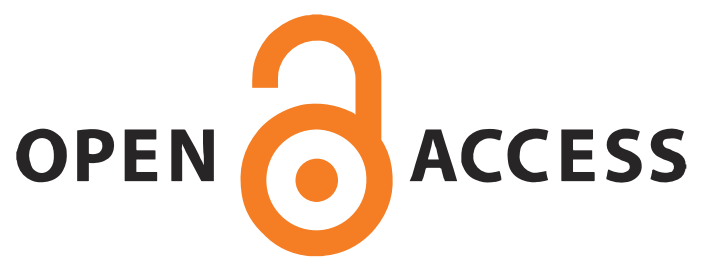




\title{
Model theory for sheaves of modules
}

\author{
Mike Prest \\ Alan Turing Building \\ School of Mathematics \\ University of Manchester \\ Manchester M13 9PL \\ UK \\ mprest@manchester.ac.uk
}

December 5, 2018

\begin{abstract}
We describe how the model theory of modules is adapted to deal with sheaves of modules.
\end{abstract}

\section{Contents}

1 Introduction 1

2 Model theory for modules $\quad 2$

3 Presheaves and sheaves of modules 4

4 Model theory for presheaves of modules $\quad 6$

5 Model theory for sheaves of modules $\quad 10$

\section{Introduction}

A sheaf may be thought of as a set of structures, indexed by the points of a topological space, which "vary in a continuous way". For example, a sheaf $\mathcal{O}$ of rings over a topological space $X$ is given by a set $\left\{\mathcal{O}_{X, x}: x \in X\right\}$ of rings (which we assume to be associative, not necessarily commutative, and each with a 1) together with a certain type of topology on the union of these sets. This is the étalé-space view of a sheaf, which we will point out after approaching the definition of a sheaf through that of a presheaf.

Sheaves arise typically in geometry, topology and analysis. Our, algebraic/modeltheoretic, interest will be in the model theory of sheaves of modules over sheaves of rings.

The model theory of modules is very well-developed and has found many applications. We will describe how to set up model theory for sheaves of modules in a way which naturally generalises how this is done for modules over a fixed 
ring (that is the case where the space $X$ has just one point). The key change is that we should regard sheaves as multi-sorted structures. The outcome is that, over topological spaces $X$ which satisfy some mild conditions, one can apply all the techniques and results of the model theory of modules.

A great deal of what we say applies to sheaves over sites (where Grothendieck topologies replace topologies in the usual sense) and to sheaves of structures other than modules but our aim is to explain the particularities of the model theory of sheaves of modules in the relatively concrete context of sheaves over topological spaces.

\section{Model theory for modules}

Here we give a very brief overview of some relevant aspects of the model theory of modules.

The model theory of modules was originally set up to deal with modules over a fixed ring $R$ (always assumed to be associative and with a 1 ). ${ }^{1}$ By default, by " $R$-module" we will mean "right $R$-module", and we will denote the category of these by Mod- $R$. Since the left $R$-modules are the right modules over the ring $R^{\text {op }}$ with the opposite multiplication, it is immaterial for the general theory whether we deal with right or left modules.

The language $\mathcal{L}_{R}$ used has a binary operation symbol + for the addition on a module, a constant symbol 0 for the zero element of a module and, for each $r \in R$, a 1-ary function symbol to express multiplication-by- $r$ on a module. In practice we use natural notation, writing the value of multiplication by $r \in R$ on an element $a$ of a module $M$ by $a r$, rather than introducting a more explicitly functional notation (such as $\left.f_{r}(a)\right)$. Also, for instance, we would write an atomic formula in variables $x_{1}, \ldots, x_{n}$ in the (simplified, using the theory of $R$-modules) form $\sum_{i=1}^{n} x_{i} r_{i}=0$. The background theory is that generated by the usual axioms for (right) $R$-modules.

The key result in the model theory of modules is pp-elimination of quantifiers (see, for example, $[8, \S 2.4]$ ).

Theorem 2.1. Let $R$ be any ring. Modulo the theory of (right) $R$-modules, every formula is equivalent to the conjunction of a sentence and finite boolean combination of pp formulas. Moreover, every sentence is a finite boolean combination of invariants conditions.

A pp (for positive primitive), also called regular, formula, is an existentially quantified conjunction of atomic formulas, that is, in our context, an existentially quantified system of $R$-linear equations (perhaps inhomogeneous equations if the formula contains parameters from a module). The solution set, $\phi(M)$, of a pp formula $\phi$, in any module $M$, is a subgroup of $M^{n}$, where $n$ is the number of free variables of $\phi$. These subgroups are the groups ppdefinable in $M$ or, as commonly said more loosely, the pp-definable subgroups of $M$. If $\phi, \psi$ are pp formulas in the same free variables, then we write $\psi \leq \phi$ if $\psi(M) \leq \phi(M)$ for every module $M$. In fact (see e.g. [9, 1.2.23]),

\footnotetext{
${ }^{1}$ One could let the ring vary by using a two-sorted language: one sort for the ring, one for the module, so that the structures are (ring, module) pairs $\left(R, M_{R}\right)$. The model theory of such pairs is, however, much less well-behaved than that for modules over a fixed ring, and not at all as amenable to useful analysis.
} 
it is enough to check this for every finitely presented module $M$, because every module is a direct limit (=directed colimit) $\lim _{\lambda} M_{\lambda}$ of finitely presented modules $M_{\lambda}$ and pp formulas commute with direct limits in the sense that $\phi\left(\underline{\lim }_{\longrightarrow} M_{\lambda}\right)=\underline{\lim }_{\lambda} \phi\left(M_{\lambda}\right)$. Recall that a module $M$ is finitely presented if it is finitely generated and finitely related, equivalently if the functor $\operatorname{Hom}_{R}(M,-)$, which we simply denote by $(M,-)$, commutes with direct limits.

An invariants condition is a sentence which says, of some pp-pair $\psi \leq \phi$, that the index of the subgroup defined by $\psi$ in that defined by $\phi$ either is less than, equal to, or greater than, $n$, for some particular integer $n$.

This partial elimination of quantifiers allowed greatly simplified proofs of much that had already been shown about the model theory of modules and it stimulated a fundamental transformation of the subject.

Elimination of quantifiers also partly explained the, already-recognised, importance (see e.g. [3], [6], [19]) of notions such as purity and pure-injectivity in the model theory of modules, where we say that an inclusion $N \rightarrow M$ of modules is pure if, for every pp formula $\phi$ in $n$ free variables $^{2}$, we have $\phi(N)=N^{n} \cap \phi(M)$. The key role of pp formulas is also seen in that they are exactly those whose solution sets are preserved by $R$-linear maps: if $f: M \rightarrow N$ is a homomorphism of $R$-modules then $f \phi(M) \leq \phi(N)$.

With elimination of quantifiers to hand, Garavaglia introduced (e.g. [5]) new ideas and connections with algebra which inspired the fundamental paper [22] of Ziegler. The area has subsequently seen yet further transformations as well as many algebraic applications, for which one may look at [8] for model theory per se and at [9] for the more algebraic/category-theoretic form of the theory and many applications. Since then, there has been further widening in viewpoint, for which one many consult [11], [12].

In a short paper one can say little of all that has been done but, for the purpose in hand, we pick out a couple of important aspects.

One is the extension of the theory to apply to multisorted modules, that is, modules over rings with many objects or, said otherwise, additive functors from a skeletally small preadditive category $\mathcal{R}$ to the category $\mathbf{A b}$ of abelian groups. This viewpoint is explained in [13] and below, in Section 4, we give the details that we will need here. A preadditive category with one object is simply a ring and an additive functor from that to $\mathbf{A b}$ is exactly a module over that ring. Essentially everything about modules, and about the model theory of modules, extends, almost without change, to the general case of such "multisorted modules". The requirement that the preadditive category $\mathcal{R}$ be skeletally small, that is, to have just a set of objects up to isomorphism, avoids set-theoretic difficulties. Model-theoretically, the change in moving from modules over rings to modules over rings with many objects is that we use a multisorted language with at least one sort for each isomorphism class of object of $\mathcal{R}$; we will see some examples later.

An important notion is that of a definable category. If $\mathcal{M}$ is the category of modules over a ring (or, more generally, over a ring with many objects), then a definable subcategory of $\mathcal{M}$ is the full subcategory on a class of modules which is closed under isomorphism, direct products, direct limits and pure submodules. We also use the term for the underlying class of objects.

Theorem 2.2. If $\mathcal{M}$ is the category of modules over a ring (or over a skeletally

\footnotetext{
${ }^{2}$ In fact, [18], see [9, 2.1.6], it is enough to check for $n=1$
} 
small preadditive category), then the following conditions are equivalent on a class $\mathcal{D}$ of modules:

(i) $\mathcal{D}$ is a definable subcategory, that is, closed in $\mathcal{M}$ under isomorphism, direct products, direct limits and pure submodules;

(ii) $\mathcal{D}$ is the class of models of a theory which is axiomatised by sentences of the form $\forall \bar{x}(\phi \rightarrow \psi)$ where $\psi, \phi$ are pp formulas (in free variables $\bar{x})$;

(iii) $\mathcal{D}$ is an axiomatisable class of modules satisfying $\mathcal{D}=\operatorname{Add}(\mathcal{D})$ (in fact $\mathcal{D}=\operatorname{add}(\mathcal{D})$ is enough).

By $\operatorname{Add}(\mathcal{D})$, respectively $\operatorname{add}(\mathcal{D})$, we mean the closure of $\mathcal{D}$ under direct summands and arbitrary, resp. finite, direct sums.

By a definable category we mean one which is equivalent to a definable subcategory of some module category. It has turned out that definable categories are the natural context for the model theory of modules, in the sense that the techniques apply, and the general results which hold for modules also hold (with minor modifications) in any definable category. Furthermore, the model theory of any definable category $\mathcal{D}$ is intrinsic, in the sense that an appropriate language and theory for which $\mathcal{D}$ is the category of models, may be defined just from the category structure of $\mathcal{D}$ (see [10, Chpt. 12]).

\section{Presheaves and sheaves of modules}

Let $X$ be a topological space. We use the notation $X$ also for the underlying set of the topology and we write $\mathrm{Op}_{X}$ for the poset of open subsets of $X$, ordered by inclusion. We can regard $\mathrm{Op}_{X}$ as a category with objects being the open sets and a (unique) arrow from $V$ to $U$ iff $V \subseteq U$. Let $\mathcal{C}$ be a category of structures for example the category of abelian groups, or rings, or commutative rings with 1 .

A presheaf $F$ of structures from $\mathcal{C}$ over $X$ is given by the following data: - for each open subset $U$ of $X$, an object $F U$ of $\mathcal{C}$;

- for each inclusion of open subsets $V \subseteq U$ of $X$, a morphism $r_{U V}^{F}=r_{U V}$ : $F U \rightarrow F V$, usually referred to as a restriction map, of $\mathcal{C}$, such that:

- for every open set $U, r_{U U}$ is the identity map id $\operatorname{id}_{F U}$ of $F U$ and

- given open sets $W \subseteq V \subseteq U$ we have $r_{U W}=r_{V W} r_{U V}$.

In other words, a presheaf in $\mathcal{C}$ over $X$ is a contravariant functor from the poset $\mathrm{Op}_{X}$, regarded as a category, to $\mathcal{C}$. From that point of view, a morphism $f: F \rightarrow G$ of presheaves is defined simply to be a natural transformation, that is, an $\mathrm{Op}_{X}$-indexed set $\left(f_{U}: F U \rightarrow G U\right)_{U}$ of morphisms of $\mathcal{C}$ such that, for every inclusion $V \subseteq U$ the following diagram commutes.

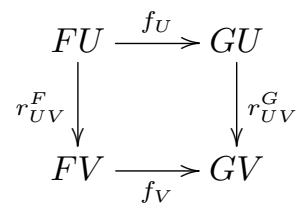

Thus we obtain the category of presheaves of $\mathcal{C}$-objects over $X$ and morphisms between them.

In particular, if $\mathcal{C}$ is the category of associative rings with 1 then we obtain the notion of a presheaf of rings. 
As a specific example, take $X$ to be the unit circle $S^{1} \subseteq \mathbb{R}^{2}$ in the real plane with its usual topology, and define a presheaf of rings by assigning, to each open subset $U$ of $S^{1}$, the $\operatorname{ring} \mathcal{C}(U, \mathbb{R})$ of continuous functions from $U$ to $\mathbb{R}$, and by assigning, to an inclusion $V \subseteq U$ of open subsets of $S^{1}$, the map from $\mathcal{C}(U, \mathbb{R})$ to $\mathcal{C}(V, \mathbb{R})$ which takes a continuous function on $U$ to its restriction to $V$. It is easily checked that this is indeed a presheaf of rings. In fact it is a sheaf in the sense of the following definition, which we state in a form which applies when the category $\mathcal{C}$ is a category of sets with structure. In that case we refer to the elements of $F U$, where $F$ is a presheaf and $U$ an open set, as sections (of $F$ ) over $U$.

A presheaf $F$ on a space $X$ is a sheaf if:

- given an open cover $U=\bigcup_{\lambda} U_{\lambda}$ of an open set $U \subseteq X$, and given, for each $\lambda$, some section $s_{\lambda} \in F U_{\lambda}$, if, for every $\lambda$, $\mu$, we have $r_{U_{\lambda}, U_{\lambda} \cap U_{\mu}}\left(s_{\lambda}\right)=$ $r_{U_{\mu}, U_{\lambda} \cap U_{\mu}}\left(s_{\mu}\right)$, then there is a section $s \in F U$ such that, for every $\lambda$, the restriction of $s$ to $U_{\lambda}$ is $s_{\lambda}$, that is, $r_{U, U_{\lambda}}(s)=s_{\lambda}$, and

- given an open cover $U=\bigcup_{\lambda} U_{\lambda}$ of an open set in $X$, and given sections $s, t \in F U$ which agree on each member of the cover - that is, if, for each $\lambda$ we have $r_{U U_{\lambda}}(s)=r_{U U_{\lambda}}(t)$ - then $s=t$.

The second condition says that sections which locally agree (that is, agree on some open cover) must be equal; in the case that the objects of $\mathcal{C}$ have an underlying abelian group structure then it is enough to take $t=0$. The first condition says that sections on a cover may be glued together to make a section on the set being covered provided that they agree on the intersections; if the second condition also holds, then there is a unique such section.

Given a presheaf $F$ over a space $X$, with values in a category $\mathcal{C}$ which has direct limits (that is, directed colimits) and given a point $x \in X$, we define the stalk of $F$ at $x$ to be $F_{x}=\lim _{U \ni x} F U$, the direct limit being taken over the directed (by intersection) system of open subsets that contain $x$. In the example above, of continuous functions on open subsets of the circle $S^{1}$, the stalk at a point $x \in S^{1}$ is the ring of so-called "germs" of continous functions at $x$.

Now we come to our main definition, that of a sheaf of modules.

Suppose that $R_{X}=(X, R)$ is a ringed space, that is a sheaf $R$ of rings (associative with 1 under our conventions) over a topological space $X$. For each open subset $U \subseteq X$ we have the ring $R U$ and the corresponding category, which we denote by Mod- $R U$, of right modules over $R U$ and for every point $x \in X$ we have the ring $R_{x}$ and its corresponding category Mod- $R_{x}$ of right modules. We will use the notations $R$ and $R_{X}$ fairly interchangably.

We define a (right) $R_{X}$-premodule to be a presheaf $M$ which assigns to each open subset $U \subseteq X$ a right $R U$-module $M U$ such that, for each inclusion $V \subseteq U$ of open subsets, the restriction $r_{U V}^{M}: M U \rightarrow M V$ is a homomorphism of $R U$-modules, where we regard $M V$ as an $R U$-module via restriction of scalars along the ring homomorphism $r_{U V}^{R_{X}}: R U \rightarrow R V$. Strictly speaking this is not, as defined, a presheaf in the sense of our earlier definition since the category where $M$ takes values varies with $U$ ! But there are ways around this - for example we could just let $M$ take values in the category of abelian groups and then add extra conditions concerning the actions of the elements of the various $R U$. In any case, it is convenient to think of the codomain category as varying. Note that the stalk $M_{x}$ at a point $x$ will be a right $R_{X, x}$-module. 
A morphism $f: M \rightarrow N$ of $R_{X}$-premodules is, if we regard $M$ and $N$ as functors, a natural transformation, that is, for each open set $U$, an $R U$-linear map $f_{U}: M U \rightarrow N U$ such that, if $V \subseteq U$ are open, then the diagram

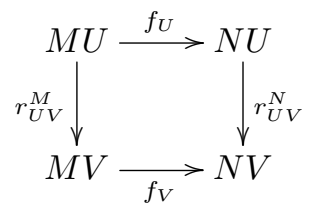

commutes. One may check that such a morphism induces, at each $x \in X$, an $R_{X, x}$-linear map $M_{x} \rightarrow N_{x}$ of stalks at $x$. These definitions give us the category PreMod- $R_{X}$ of $R_{X}$-premodules. It is an abelian ${ }^{3}$ category and, is in fact, Grothendieck and locally finitely presented. We will examine the reason for the latter since it leads directly to setting up the model theory of such structures. We denote by Mod- $R_{X}$ the category of sheaves of modules - the full subcategory of PreMod- $R_{X}$ with objects those presheaves of modules which are actually sheaves. This also is abelian and Grothendieck, but is not always locally finitely presented, though in many important cases it is.

Bundling together the stalks of a sheaf gives an alternative view of a sheaf. Given a sheaf $F$, we form the disjoint union of the stalks $F_{x}$, for $x \in X$ of $F$. This union is then given a topology which locally looks like that of $X$ (see, e.g., [21] for details) and the sections $F U$ of $F$ at an open set $U$ then become the continuous maps $s$, from $U$ to the resulting étalé space, which are such that $\pi s=\mathrm{id}_{U}$ where $\pi$ is the projection map from the étalé space to $X$ which takes an element of $F_{x}$ to the point $x$ (and $\operatorname{id}_{U}$ is the identity map on $U$ ). One more piece of notation: if $F$ is a sheaf of additive structures then we define the support of a section $s \in F U$ to be the set, $\operatorname{supp}(s)=\left\{x \in X: s_{x} \neq 0\right\}$, of points where the image $s_{x}$ of $s$ in $F_{x}$, under the natural map $F U \rightarrow F_{x}$, is nonzero.

\section{Model theory for presheaves of modules}

A category $\mathcal{C}$ is said to be finitely accessible (see [1]) if it has direct limits, if there is, up to isomorphism, just a set of finitely presented objects of $\mathcal{C}$ and if every object of $\mathcal{C}$ is a direct limit of finitely presented objects, where an object $A \in \mathcal{C}$ is finitely presented if the functor $\mathcal{C}(A,-)$, which we abbreviate as $(A,-)$, commutes with direct limits. This is equivalent to the object $A$ being "finitely generated and finitely related" in $\mathcal{C}$ if those terms make sense in $\mathcal{C}$ (for instance, if $\mathcal{C}$ is the category of rings, or of groups, or of modules over a ring).

To expand on the condition that the representable functor $(A,-)$ commute with direct limits, this means that, given any directed system, $\left(\left(C_{\lambda}\right)_{\lambda \in \Lambda},\left(f_{\lambda \mu}\right.\right.$ : $\left.\left.C_{\lambda} \rightarrow C_{\mu}\right)_{\lambda \leq \mu \in \Lambda}\right)$ with direct limit $\left(C,\left(f_{\lambda \infty}: C_{\lambda} \rightarrow C\right)_{\lambda}\right)$ - so $f_{\mu \infty} f_{\lambda \mu}=f_{\lambda \infty}$ for all $\lambda \leq \mu$ - and given any $g: A \rightarrow C$, there is $\lambda^{\prime}$ and $g^{\prime}: A \rightarrow C_{\lambda^{\prime}}$ such that $f_{\lambda^{\prime} \infty} g^{\prime}=g$. Moreover, such a factorisation must be essentially unique in the sense that, if also there is $\lambda^{\prime \prime}$ and $g^{\prime \prime}: A \rightarrow C_{\lambda^{\prime \prime}}$ such that $f_{\lambda^{\prime \prime} \infty} g^{\prime \prime}=g$, then there is $\mu \geq \lambda^{\prime}, \lambda^{\prime \prime}$ such that $f_{\lambda^{\prime} \mu} g^{\prime}=f_{\lambda^{\prime \prime} \mu} g^{\prime \prime}$.

We denote by $\mathcal{C}^{\text {fp }}$ the full subcategory of finitely presented objects of $\mathcal{C}$. If

\footnotetext{
${ }^{3}$ We will not present background on abelian category theory here but there are many suitable references, for example [4], [20].
} 
$\mathcal{C}$ is finitely accessible and both complete and cocomplete then it is a locally finitely presented category. Both the category all presheaves and of all sheaves of modules over a ringed spaces are complete and cocomplete, so we will use the terms finitely accessible and locally finitely presented interchangably for these.

The fact that, for any ringed space $R_{X}$, PreMod- $R_{X}$ is locally finitely presented is a special case of a general fact for functor categories and, as in that general case, it is the representable functors which provide a generating collection (a set up to isomorphism since $\mathrm{Op}_{X}$ has just a set of objects) of finitely presented presheaves. We describe these representable functors in specific presheaf terms.

Let $U \in \mathrm{Op}_{X}$ be any open subset of $X$ and let $j: U \rightarrow X$ denote the inclusion map. We define the presheaf $j_{0} R_{U}$ as follows (we will explain the notation after that):

$$
j_{0} R_{U}(V)=\left\{\begin{array}{ll}
R(V) & \text { if } V \subseteq U \\
0 & \text { otherwise }
\end{array} .\right.
$$

Here $j_{0}$ denotes a functor from PreMod- $R_{U}$ to PreMod- $R_{X}$ and $R_{U}$ denotes the restriction, $\left.R_{X}\right|_{U}$, of $R_{X}$ to $U$. For any presheaf $F$ on a space $X$ and open subset $U$ of $X$, the restriction of $F$ to $U$ is the presheaf $\left.F\right|_{U}$ on $U$ which is given by $\left.F\right|_{U} V=F V$ for $V$ an open subset of $U .{ }^{4}$ It is direct from the definition that the restriction of a sheaf is again a sheaf. For any $G \in \operatorname{PreMod}-R_{U}$ the presheaf $j_{0}$ defined by $j_{0} G \cdot V=\left\{\begin{array}{ll}G V & \text { if } V \subseteq U \\ 0 & \text { otherwise }\end{array}\right.$ is the extension by 0 of $G$ (to a presheaf on $X$ ).

Proposition 4.1. (e.g. [2, p. 7 Prop. 6]) If $R_{X}$ is any ringed space, then the category PreMod- $R_{X}$ of presheaves of $R_{X}$-modules is abelian Grothendieck and locally finitely presented, with the $j_{0} R_{U}$, for $U \in \mathrm{Op}_{X}$ being a generating set of finitely presented objects.

By a generating set $\mathcal{G}$ of objects of an Grothendieck abelian category $\mathcal{C}$ we mean that for every object $C \in \mathcal{C}$ there is an exact sequence $H^{\prime} \rightarrow H \rightarrow C \rightarrow 0$ where $H^{\prime}, H$ are direct sums (possibly infinite) of copies of objects in $\mathcal{G}$. We do not mean that it is lim-generating (in the sense of the definition of finitely accessible category). But, if $\mathcal{G}$ is a set of finitely presented objects which is generating in the sense just defined, then $C \in \mathcal{C}$ will be finitely presented iff there is such a presentation where both $H^{\prime}$ and $H$ are finite direct sums of copies of objects in $\mathcal{G}$ and it is the case that the collection of finitely presented objects of $\mathcal{C}$ will be lim-generating in $\mathcal{C}$, so $\mathcal{C}$ will be finitely accessible (indeed locally finitely presented).

The basic idea for setting up (finitary) model theory in any finitely accessible category (with products) $\mathcal{C}$ is that, since every object $C \in \mathcal{C}$ is determined by the morphisms to it from finitely presented objects, we take these morphisms to be the "elements" of $C$. But morphisms with different domains should be elements of different kinds - formally of different sorts. This means that the formal language we set up is naturally multisorted, with one sort, $\sigma_{A}$ say, for each finitely presented object $A$ of $\mathcal{C}$ and with the elements of $C \in \mathcal{C}$ of sort $\sigma_{A}$ being the elements of the set, $(A, C)$, of morphisms from $A$ to $C$. We should

\footnotetext{
${ }^{4}$ In category-theoretic terms it is the restriction of the contravariant functor $F$ to the full subcategory on the objects with a morphism to $U$.
} 
use a set $\mathcal{G}$ (rather than a proper class) of sorts, so we restrict $A$ to range over some set of finitely presented objects which contains at least one copy (to isomorphism) of each finitely presented object. This gives us the sorts of our language for $\mathcal{C}$. It does not depend, in any way that matters, on the actual choice of set of finitely presented objects that we use, as long as it has a copy of each (or indeed, "enough") of the finitely presented objects.

We also introduce function symbols, one for each morphism between objects in our chosen set $\mathcal{G}$ of finitely presented objects. If $f: A \rightarrow B$ is such a morphism, then the corresponding function symbol, for which we will use the same symbol $f$, has domain $\sigma_{B}$ and codomain $\sigma_{A}$, reflecting the direction of the induced morphism $(f,-):(B,-) \rightarrow(A,-)$, given by $g \mapsto g f$ (where $f \in(B, C)$ for any $C \in \mathcal{C})$, between representable functors.

This viewpoint on what are the "elements" of a structure might seem unfamilar but it is exactly what is seen in the basic fact from module theory that a module $M$ over a ring $R$ is isomorphic to the module ${ }^{5}$ of homomorphisms $\left(R_{R}, M\right)$ from $R_{R}$, meaning $R$ regarded as a right $R$-module, to $M$. That is because each homomorphism is determined by the image of 1 and every element of $M$ is such an image.

For example, under this expanded viewpoint, if we take a direct product $R^{(n)}$ of copies of $R_{R}$, then the "elements" of a module $M$ of sort $R^{(n)}$ are exactly the $n$-tuples of elements of $M$. More generally, if $A$ is a finitely presented module, then the elements of a module $M$ in sort $A$ (that is, of $\sigma_{A} M$ ) could be regarded as the $n$-tuples (if $A$ is $n$-generated) of elements of $M$ which satisfy certain $R$-linear relations, namely those which generate all the $R$-linear relations on a chosen generating set of $n$ elements for $A$.

This example of modules also shows that it is not necessary to represent every isomorphism class of finitely presented object when setting up the language it is enough to have a set of sorts corresponding to a set of finitely presented objects which generate the category in the sense defined after Proposition 4.1 (that refers to the special case of a Grothendieck abelian category, but it is such categories with which we will be concerned). Any two such languages set up using generating sets will be inter-interpretable (each formula in the one language can be translated to an equivalent formula in the other), so will give the same model theory for the structures in $\mathcal{C}$. In the case of categories, PreMod- $R_{X}$, of presheaves we will use the representable functors - the extensions by 0 - defined above. Let us return now to this case.

The presheaves $j_{0} R_{U}$ as $U$ ranges over subsets of $X$ form a generating set of finitely presented objects of PreMod- $R_{X}$, so we will use these, hence the open subsets of $X$, to index the sorts of our language for PreMod- $R_{X}$. We should describe the functors $\left(j_{0} R_{U},-\right)$ and the morphisms between them in order to understand something of what can be expressed in this language.

Lemma 4.2. Suppose that $R_{X}$ is a ringed space. Let $j: U \rightarrow X$ be the inclusion of an open subset in $X$, let $F \in \operatorname{PreMod}-R_{U}$ and let $G \in \operatorname{PreMod}-R_{X}$. Then there is a natural isomorphism of groups $\left(j_{0} F, G\right) \simeq\left(F,\left.G\right|_{U}\right)$.

If $U$ is an open subset of $X$, then the functor $\left(j_{0} R_{U},-\right)$ is, in the view of presheaves as contravariant functors on $\mathrm{Op}_{X}$, the representable functor corresponding to $U \in \mathrm{Op}_{X}$ hence, by the Yoneda Lemma, $\left(j_{0} R_{U}, G\right) \simeq G U$. In

\footnotetext{
${ }^{5}$ It is a right module via the left action of $R$ on the module $R_{R}$.
} 
particular, if $U, W$ are arbitrary open subsets of $X$, then $\left(j_{0} R_{U}, j_{0} R_{W}\right) \simeq$ $j_{0} R_{W} \cdot U=\left\{\begin{array}{ll}R_{X} U & \text { if } U \subseteq W \\ 0 & \text { otherwise }\end{array}\right.$. We can understand this application of the Yoneda Lemma more algebraically by noting that $j_{0} R_{U}$ is generated by the section $1_{U} \in j_{0} R_{U} \cdot U=R_{X} U$, in the sense that, for every open subset $V$ of $U$, the image, $r_{U V}\left(1_{U}\right)$, of $1_{U}$ under the restriction map from $U$ to $V$, is equal to $1_{V} \in R_{X} V$, which generates $R_{X} V=j_{0} R_{U} \cdot V$ as an $R_{X} V$-module. Therefore any morphism $f:\left.j_{0} R_{U} \rightarrow G\right|_{U}$ will be determined by the image $f_{U}\left(1_{U}\right) \in G U$ where $f_{U}$ is the component of $f$ at $U$.

Thus, if we use the language for PreMod- $R_{X}$ based on the generating set $\left(j_{0} R_{U}\right)_{U \in \mathrm{Op}_{X}}$, then the function symbols of the language, beyond those used to express the abelian group structure of each sort $\sigma_{U}=\left(j_{0} R_{U},-\right)$ are as follows: given open subsets $U, W$ of $X$ then, if $U \subseteq W$ the function symbols from sort $\sigma_{W}$ to sort $\sigma_{U}$ are naturally indexed by the elements of $R_{X} U$, otherwise there is only the zero function symbol from $\sigma_{W}$ to $\sigma_{U}$.

Using the Yoneda Lemma as above, we can explicitly describe the interpretations of these function symbols, as follows.

Given open sets $U \subseteq W$ and $t \in R_{X} U$, regarded (as above) as an element of $j_{0} R_{W} \cdot U$, hence as a morphism from $j_{0} R_{U}$ to $j_{0} R_{W}$, and given any $G \in$ PreMod- $R_{X}$, we have the following diagram in PreMod- $R_{X}$ showing the action of $t$ :

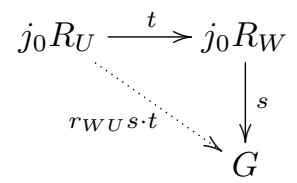

namely $t:\left(j_{0} R_{W}, G\right)=G W=\sigma_{W} G \rightarrow\left(j_{0} R_{U}, G\right)=G U=\sigma_{U} G$, takes a section $s \in G W$ to its restriction to $U$ followed by multiplication by $t$ - the result is a section in $G U$.

We can regard these actions, as multiplications by elements of a ring with many objects (see [13], also [12], for this point of view). Thus the $R_{X}$-presheaves become "modules over a ring with many objects" and, in fact (see [13] for an overview, [9] for details) the model theory of modules over the usual, 1-sorted, rings is applicable in its entirety. Let us give some examples (also see [14] and [17]) of what one can say with this language. After that, we will move on to the category of $R_{X}$-sheaves.

First, a notational point. If $V \subseteq U$ are open then, in any presheaf, $F$, the restriction map $r_{U V}^{F}$ is the interpretation of a function symbol $r$ (from sort $\sigma_{U}$ to $\left.\sigma_{V}\right)$ in the language. The functional notation for the value of this map on a section $a \in F U$ is $r_{U V}(a)$, whereas the right module notation is $a r$. In practice, since perhaps the meaning is clearer, we shall use the former, functional notation, but bear in mind that it is naturally written in the module language as a right multiplication.

A presheaf $F$ is said to be separated if, for every open set $U$, open cover $\left(U_{\lambda}\right)_{\lambda}$ of $U$ and sections $s, t \in F U$, if, for every $\lambda$ we have $r_{U U_{\lambda}}(s)=r_{U U_{\lambda}}(t)$ then $s=t$ (that is, if sections agree locally, then they are equal). Of course in the additive situation, it is enough to consider the case where $t=0$. Given such an open set and open cover of it, note that there is a possibly infinitary 
sentence -

$$
\forall x, y\left(\left(\bigwedge_{\lambda} r_{U U_{\lambda}}(x)=r_{U U_{\lambda}}(y)\right) \rightarrow(x=y)\right)
$$

- which expresses this condition (where the variables have sort $\sigma_{U}$ ) If the cover is, or may be taken to be, finite (so, in particular if $U$ is compact) then this will be, or be equivalent to, a sentence of finitary model theory. As we let these sentences range over all open sets and open covers (finite if possible), then we see that the property of being a separated presheaf is expressible in an infinitary version of our language, finitary if every open set is compact (that is, if the space is noetherian). We deduce that over any noetherian space the category of separated presheaves is definable in the sense discussed in Section 2.

The other condition necessary for a presheaf $F$ to be a sheaf is that, given any open set $U$, open cover $\left(U_{\lambda}\right)_{\lambda}$ of $U$ and set $\left(s_{\lambda} \in F U_{\lambda}\right)_{\lambda}$ of compatible sections (meaning that, for every $\lambda, \mu, r_{U_{\lambda}, U_{\lambda} \cap U_{\mu}}^{F}\left(s_{\lambda}\right)=r_{U_{\mu}, U_{\lambda} \cap U_{\mu}}^{F}\left(s_{\mu}\right)$ ), there is $s \in F U$ such that $r_{U U_{\lambda}}^{F}(s)=s_{\lambda}$ for every $\lambda$. We see that this can be expressed by the sentence, infinitary if the cover is infinite,

$$
\left.\forall\left(x_{\lambda}\right)_{\lambda}\left(\bigwedge_{\lambda \mu} r_{U_{\lambda}, U_{\lambda} \cap U_{\mu}}\left(x_{\lambda}\right)=r_{U_{\mu}, U_{\lambda} \cap U_{\mu}}\left(x_{\mu}\right)\right) \rightarrow\left(\exists x \bigwedge_{\lambda} r_{U U_{\lambda}}(x)=x_{\lambda}\right)\right)
$$

(where the variable $x_{\lambda}$ has sort $\sigma_{\lambda}$ and $x$ has sort $\sigma_{U}$ ). These sentences, ranging over open sets and covers, therefore axiomatise the glueing property. Combining this with the observations on the separation property, we deduce that over any noetherian space the category of sheaves is a definable subcategory of the category of presheaves (one may also prove the converse, so this characterises noetherian spaces - see $[15,3.12])$. But we will see in the next section that it is possible for the category of sheaves to be definable (that is a definable subcategory of some category of multisorted modules), without necessarily being a definable subcategory of the category of presheaves.

For another example of what can be expressed using this language, if $U$ is any open set then the (closure under isomorphism) of the class of presheaves of the form $j_{0} F$ for some $F \in \operatorname{PreMod}-R_{U}$ is axiomatised by the set of sentences of the form $\forall x_{V}\left(x_{V}=0\right)$ where $V$ ranges over the open sets which are not contained in $U$ (and the notation $x_{V}$ indicates that $x$ is a variable of sort $\sigma_{V}$ ).

As yet another type of example, the constant presheaves (those such that each restriction map is an isomorphism) are axiomatised by the set of sentences of the form $\left(\forall y \exists x\left(y=r_{U V}(x)\right)\right) \wedge\left(\forall x\left(r_{U V}(x)=0_{V} \rightarrow x=0_{U}\right)\right)$ as $V, U$ range over open subsets with $V \subseteq U$, where $x$ has sort $\sigma_{U}, y$ has sort $\sigma_{V}$ and the subscripts on the constant symbols 0 indicate their sort.

\section{$5 \quad$ Model theory for sheaves of modules}

There is a canonical functor, sheafification, which turns each presheaf $F$ into the sheaf $a F$ which best approximates it in the category of sheaves. More precisely, sheafification is left adjoint to the forgetful functor (that is, the inclusion) $u: \operatorname{Mod}-R_{X} \rightarrow$ PreMod- $R_{X}$. So for every $F \in \operatorname{PreMod}-R_{X}$ and $M \in \operatorname{Mod}-R_{X}$ there are natural isomorphisms $(F, u M) \simeq(a F, M)$. Roughly, $a F$ is formed from $F$ by first identifying every two sections which agree on some open cover, so as to obtain a separated presheaf, then adding, as new sections, the results 
of glueing together compatible families of sections. In the context of presheaves of modules, sheafification is localisation in the sense of Gabriel (see [20]). For, the presheaves $F$ such that, for every $U \in \mathrm{Op}_{X}$ and section $s \in F U$, there is an open cover of $U$ such that each restriction of $s$ is zero, are exactly those whose sheafification $a F=0$, and these form a hereditary torsion class of presheaves, localisation with respect to which is the sheafification functor.

In the particular case where the space $X$ is noetherian, this is a finite-type localisation $[15,3.8]$, which has the consequence that the sheaves form an axiomatisable, indeed, definable, subcategory of PreMod- $R_{X}[15,3.12]$. In that case, therefore, the language for presheaves described above also may be used for developing the model theory of $\operatorname{Mod}-R_{X}$ since the objects in the latter category form an elementary class of presheaves. But it is not necessary that $X$ be noetherian in order to have a good model theory for sheaves; a basis $\mathcal{B}$ of compact open sets closed under intersection is enough $[16,3.3]$ since, in that case, the category Mod- $R_{X}$ of sheaves is locally finitely presented $[16,3.5]$. That is, rather than indexing the sorts of the language using all open sets, it is enough to use those from $\mathcal{B}$. Because we are using finitary model theory, we need such a basis consisting of compact open sets. That is because our "elements" of sorts of sheaves - that is, sections over open sets in the basis should be "finitary elements", meaning that if such an element belongs to a directed sum or union, then it belongs to some member of that sum or union. Having just a basis of compact sets is enough because sections are locally determined. The requirement that $\mathcal{B}$ be closed under intersection enables us to write the compatibility-of-sections condition in the resulting formal language. We give some details, but quite briefly since they are very similar to those seen for presheaves.

Suppose then that $\mathcal{B}$ is a basis, closed under intersection, of open sets for the topology on $X$. Then it turns out that the sheaves $j_{!} R_{U}$ for $U \in \mathcal{B}$ form a generating set of finitely presented objects of Mod- $R_{X}$. Here $j_{!} R_{U}$ denotes the sheafification, $a\left(j_{0} R_{U}\right)$, of $j_{0} R_{U}$; it is the sheaf extension by 0 of $R_{U}$ to $X$. In general, if $G \in \operatorname{Mod}-R_{U}$ then its sheaf extension, $j_{!} G$, by 0 to $X$ may be defined by

$$
j_{!} G(V)=\{s \in G(V \cap U): \operatorname{supp}(s) \text { is closed in } V\} .
$$

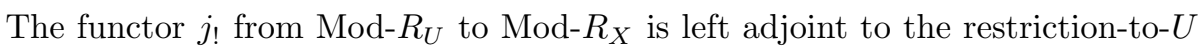
functor, so $\left(j_{!} G, F\right) \simeq\left(G,\left.F\right|_{U}\right)$ for $G \in \operatorname{Mod}-R_{U}$ and $F \in \operatorname{Mod}-R_{X}$, and $j !$ is an equivalence between Mod- $R_{U}$ and the subcategory of Mod- $R_{X}$ consisting of the sheaves which have support contained in $U$ (see [7, pp. 106/7]). Thus the $j_{!} R_{U}$ play a very similar role in Mod- $R_{X}$ to that played in PreMod- $R_{X}$ by the $j_{0} R_{U}$. But, in contrast to the presheaves $j_{0} R_{U}$, they are not necessarily finitely presented. However:

Proposition 5.1. ([16, 3.7]) If $X$ has a basis, closed under intersection, of compact open subsets and if $U$ is compact open, then $j_{!} R_{U}$ is a finitely presented sheaf.

Furthermore, we don't need all the $j_{!} R_{U}$ in order to generate $\operatorname{Mod}-R_{X}$ :

Proposition 5.2. ([16, 3.2]) If $\mathcal{B}$ is a basis of open subsets for the topology on $X$ then the $j_{!} R_{U}$ for $U \in \mathcal{B}$ together generate Mod- $R_{X}$. 
Corollary 5.3. ([16, 3.5]) If $\left(X, R_{X}\right)$ is a ringed space and if $\mathcal{B}$ is a basis, closed under intersection, of compact open subsets of $X$, then the category Mod- $R_{X}$ of $R_{X}$-modules is locally finitely presented, with the $j_{!} R_{U}$, for $U \in \mathcal{B}$ forming a generating set of finitely presented objects.

In that case therefore, having fixed such a basis $\mathcal{B}$, what we have said about the multisorted model theory for a locally finitely presented category applies, to give us a language $\mathcal{L}_{\mathcal{B}}$ for $R_{X}$-modules. This language has a sort for each $U \in \mathcal{B}$ and a function symbol for each morphism in each $\left(j_{!} R_{U}, j_{!} R_{V}\right)$ with $U, V \in \mathcal{B}$. Given any $F \in \operatorname{Mod}-R_{X}$, the resulting $\mathcal{L}_{\mathcal{B}}$-structure has value $\left(j_{!} R_{U}, F\right) \simeq F U$ in sort $\sigma_{U}$ and the description of the interpretations of the function symbols of $\mathcal{L}_{\mathcal{B}}$ as maps between these sorts is similar to that for presheaves. Namely ([14, p. 1189, 1.4]) the elements of $\left(j ! R_{U}, j_{!} R_{V}\right)$ may be identified with the sections $r$ of $R_{U \cap V}$ which have support closed in $U$ and the action of such $r$ (that is, the interpretation of the corresponding function symbol on a sheaf $F$ ), regarded as a map from $F V$ to $F U$, is restriction from $F V$ to $F(U \cap V)$, followed by multiplication by $r$ regarded as an element of $R_{U \cap V}$, followed by inclusion in $F U$.

We remark that in many examples, in particular those typically seen in algebraic geometry, it will be the case that the underlying space $X$ has a basis of compact open sets which is closed under intersection, hence the category $\operatorname{Mod}-R_{X}$ will be locally finitely presented and we will have a good, finitary, model theory of sheaves. Indeed, in many examples, every open set will be compact, therefore sections over any open set can be referred to by variables in our language.

One can see a variety of examples of what can be expressed about sheaves using this language in [14], where strongly minimal sheaves are considered and there is some comparison of stalkwise, local and global properties. There are also many examples of definable subcategories (and definable $=$ interpretation functors between them) in the recent paper [17], though that paper uses alternative, algebraic, characterisations of these concepts rather than explicitly introducing the formal language.

\section{References}

[1] J. Adámek and J. Rosický, Locally Presentable and Accessible Categories, London Math. Soc. Lecture Note Ser., Vol. 189, Cambridge University Press, 1994.

[2] F. Borceux and G. Van den Bossche, Algebra in a Localic Topos with Applications to Ring Theory, Lecture Notes in Mathematics, Vol. 1038, Springer-Verlag, 1983.

[3] P. Eklof and G. Sabbagh, Model-completions and modules, Ann. Math. Logic, 2(3) (1971), 251-295.

[4] P. Freyd, Abelian Categories, Harper and Row, 1964.

[5] S. Garavaglia, Dimension and rank in the model theory of modules, preprint, University of Michigan, 1979, revised 1980. 
[6] L. Gruson and C. U. Jensen, Modules algébriquement compact et foncteurs $\varliminf^{(i)}$, C. R. Acad. Sci. Paris, 276 (1973), 1651-1653.

[7] B. Iversen, Cohomology of Sheaves, Springer-Verlag, 1986.

[8] M. Prest, Model Theory and Modules, London Math. Soc. Lect. Note Ser., Vol. 130, Cambridge University Press, 1988.

[9] M. Prest, Purity, Spectra and Localisation, Encyclopedia of Mathematics and its Applications, Vol. 121, Cambridge University Press, 2009.

[10] M. Prest, Definable additive categories: purity and model theory, Mem. Amer. Math. Soc., Vol. 210/No. 987, 2011.

[11] M. Prest, Abelian categories and definable additive categories, arXiv:1202.0426.

[12] M. Prest, Modules as exact functors, Proceedings of 2016 Auslander Distinguished Lectures and Conference, Contemporary Mathematics, Vol. 716, Amer. Math. Soc., to appear. arXiv:1801.08015

[13] M. Prest, Multisorted modules and their model theory, Contemporary Mathematics, to appear. arXiv:1807.11889

[14] M. Prest, V. Puninskaya and A. Ralph, Some model theory of sheaves of modules, J. Symbolic Logic, 69(4) (2004), 1187-1199.

[15] M. Prest and A. Ralph, On sheafification of modules, preprint, University of Manchester, 2001, revised 2004, available at http://www.maths . manchester.ac.uk/ mprest/publications.html

[16] M. Prest and A. Ralph, Locally finitely presented categories of sheaves of modules, preprint, University of Manchester, 2001, revised 2004 and 2018, available at http://www.maths.manchester.ac.uk/ mprest/publications.html

[17] M. Prest and A. Slávik, Purity in categories of sheaves, preprint, 2018, arXiv:1809.08981.

[18] Ph. Rothmaler, A trivial remark on purity, p. 127 in Proc. 9th Easter Conf. on Model Theory, Gosen 1991, Seminarber. 112, Humboldt-Univ. zu Berlin, 1991.

[19] G. Sabbagh, Sous-modules purs, existentiellement clos et élementaires, C. R. Acad. Sci. Paris, 272 (1971), 1289-1292.

[20] B. Stenström, Rings of Quotients, Springer-Verlag, 1975.

[21] B. R. Tennison, Sheaf Theory, London Mathematical Society Lecture Note Ser., Vol. 20, Cambridge University Press, 1975.

[22] M. Ziegler, Model theory of modules, Ann. Pure Appl. Logic, 26(2) (1984), 149-213. 